\title{
Microstructure of Pyramidal Defects in InSb Layers Grown by Atomic Layer Molecular Beam Epitaxy on InP Substrates
}

\author{
J.C. Ferrer $\left({ }^{1,2, *}\right)$, F. Peiró $\left({ }^{1}\right)$, A. Cornet $\left({ }^{1}\right)$, J.R. Morante $\left({ }^{1}\right)$, T. Utzmeier $\left({ }^{3}\right)$ \\ and F. Briones $\left({ }^{3}\right)$ \\ ( ${ }^{1}$ EME, Dept Física Aplıcada 1 Electrònica, Universitat de Barcelona, Av. Diagonal, 645-647, \\ 08028 , Barcelona, Spain \\ $\left({ }^{2}\right)$ Serveis Científico-Tècnics, Universıtat de Barcelona, Lluís Solé i Sabarís, 1-3, \\ 08028, Barcelona, Spain \\ $\left(^{3}\right)$ Instituto de Microelectrónica de Madrid (CSIC), Isaac Newton, 8, Parque Tecnológıco de \\ Madrid, 28760, Tres Cantos, Madrid, Spain
}

(Received 3 October 1996, revised 22 April 1997, accepted 25 August 1997)

PACS.61.72.-y - Defects and impurities in crystals, microstructure

PACS.68.55.-a - Thin film structure and morphology

PACS 61.16.-d - Electron, ion and scanning probe microscopy

\begin{abstract}
We report on the structural characterization of epitaxial InSb films grown on InP substrates by atomic layer molecular beam epitaxy at relatively low temperatures $\left(330{ }^{\circ} \mathrm{C}<T<400{ }^{\circ} \mathrm{C}\right)$. Moreover, we study the effect of the introduction of an intermediate InSb/InP buffer layer grown by molecular beam epitaxy. The studies were carried out by TEM and HRTEM, to investigate the densities and nature of the defects and the accommodation mechanism between the two types of layers which have a large lattice mismatch $(10.4 \%)$. Results show a high defect density at the interface vicinity whatever the growth method employed, with or without buffer layers, but better quality layers are obtained as growth proceeds. The prevailing type of defects are threading dislocations and stacking faults for both types of samples, but the introduction of the intermediate layers leads to the formation of two types of complex three-dimensional defects, consisting in crystal misorientations, that induce an anomalous growth of the InSb layer leading to different growth rates and the formation of pyramidal or truncated pyramidal hillocks on the surface. In this case scanning electron microscopy and Raman analysis were also performed to study the influence of the defects on surface morphology and confirm their structure. Moreover, anisotropy of the stacking fault distribution is noticed in this sample: the density for [1]10]-(111)A slp planes is higher than for the [110]-(111)B slip planes. Strain due to large lattice mismatch is relieved in both types of samples by the generation of a pure edge-type misfit dislocation array.
\end{abstract}

\section{Introduction}

Epitaxially grown InSb layers have been of interest in recent years due to their potential applications to the fabrication of low-dimensional structures. The interest of the InSb comes from

$\left(^{*}\right)$ Author for correspondence (e-mail cornet@iris1.fae.ub es) 
Table I. - Growth parameters and defect densities of analyzed samples.

\begin{tabular}{|c|c|c|c|c|c|c|}
\hline Sample & $\begin{array}{c}\text { Growth } \\
\text { temperature } \\
\left({ }^{\circ} \mathrm{C}\right)\end{array}$ & $\begin{array}{c}\text { Buffer } \\
\text { layers }\end{array}$ & $\begin{array}{c}\text { Thickness } \\
(\mu \mathrm{m})\end{array}$ & $\begin{array}{c}\text { Threading } \\
\text { dislocation } \\
\rho_{\mathrm{TD}}\left(\mathrm{cm}^{-2}\right)\end{array}$ & $\begin{array}{c}\text { Stacking } \\
\text { fault } \\
\rho_{\mathrm{SF}}\left(\mathrm{cm}^{-2}\right)\end{array}$ & $\begin{array}{c}\text { Complex } \\
\text { twins } \\
\rho_{\mathrm{CT}}\left(\mathrm{cm}^{-2}\right)\end{array}$ \\
\hline $\mathrm{A} 1$ & 350 & - & 300 & $14 \times 10^{7}$ & $<10^{5}$ & - \\
$\mathrm{A} 2$ & 365 & - & 1.30 & $4.6 \times 10^{8}$ & $<10^{5}$ & - \\
$\mathrm{A} 3$ & 400 & - & 1.35 & $4.0 \times 10^{8}$ & $25 \times 10^{6}$ & - \\
$\mathrm{B}$ & 330 & InSb (upper, $\left.365^{\circ} \mathrm{C}\right)$ & 1.50 & $6.8 \times 10^{7}$ & $1.2 \times 10^{7}[110]$ & $50 \times 10^{5}$ (I-type) \\
& & InP (lower, $\left.420^{\circ} \mathrm{C}\right)$ & & & $5.5 \times 10^{6}[110]$ & $2.2 \times 10^{6}$ (II-type) \\
\hline
\end{tabular}

its low band-gap energy that allows it to be used as material for infrared sources and detectors. However, the conduction of InSb substrates is too high even when they are cooled to $77 \mathrm{~K}$ or $4 \mathrm{~K}$ to make them of use for homoepitaxial growth. Thus the investigation of heteroepitaxial growth on mismatched substrates is necessary. Several reports have been published recently dealing with the structural characterization, electrical and optical properties, and epitaxial deposition techniques of InSb on highly mismatched substrates like GaAs or InP grown by molecular beam epitaxy (MBE) [1-4]. In this paper we report on the morphology of InSb layers grown by atomic layer molecular beam epitaxy (ALMBE) and the role played by a MBE $\mathrm{InSb} / \mathrm{InP}$ buffer layer. We show that the strain relaxation by a misfit dislocation array and the accumulation of defects in the region near the interface make the deposition of InSb on InP substrates an excellent way to grow high quality material although a high lattice mismatch of $10.4 \%$ is present.

\section{Experimental}

Samples were grown by atomic layer molecular beam epitaxy (ALMBE) in a conventional solid-source MBE [6,7] system on semi-isolating (001) InP substrates. Typical beam equivalent pressure $(\mathrm{BEP})$ was 1.5 to $3 \times 10^{-6}$ torr, with $\mathrm{BEP}\left(\mathrm{Sb}_{4}\right) / \mathrm{BEP}(\mathrm{In}) \sim 1.6$. For the $\mathrm{ALMBE}$ growth we found better growth conditions for relatively long $\mathrm{Sb}$ opening intervals. The stoichiometric composition of the layers was controlled by means of reflectance difference (RD) measurements of the surface In-dimers fraction at a wavelength of $1 \mu \mathrm{m}$. Growth velocities were calibrated by reflection high energy electron diffraction (RHEED) oscillations. After oxide desorption at $490{ }^{\circ} \mathrm{C}$ the $\mathrm{InSb}$ layers were grown at temperatures $T_{\mathrm{g}}$ between 300 and $400^{\circ} \mathrm{C}$ up to a total thickness of $3 \mu \mathrm{m}$ After approximately $1.5 \mathrm{ML}$ 's of InSb growth we observed the transition from two-dimensional (2D) to the three-dimensional (3D) island growth, indicated by a spotty RHEED pattern. After further $40 \mathrm{ML}$ 's a plain growth front could be recovered. Growth temperatures and layer structures are presented in Table I. Three samples (called A1-A3) without buffer layer, grown by ALMBE at different temperatures and thicknesses and another one (called B) with an InSb/InP MBE buffer layer were grown in order to study the role of these technological parameters.

The studies of morphology and defect structure were carried out by transmission electron microscopy (TEM) and high resolution transmission electron microscopy (HRTEM) with a Philips CM 30 operated at $300 \mathrm{kV}$. The densities of the different defect types were measured and used to asses the mechanism of strain relaxation. Samples were prepared by conventional mechanical polishing procedures, and final Ar ion milling with $\mathrm{N}_{2}$ cooling, until perforation for both plan view and cross section configurations Scanning electron microscopy (SEM) in a 

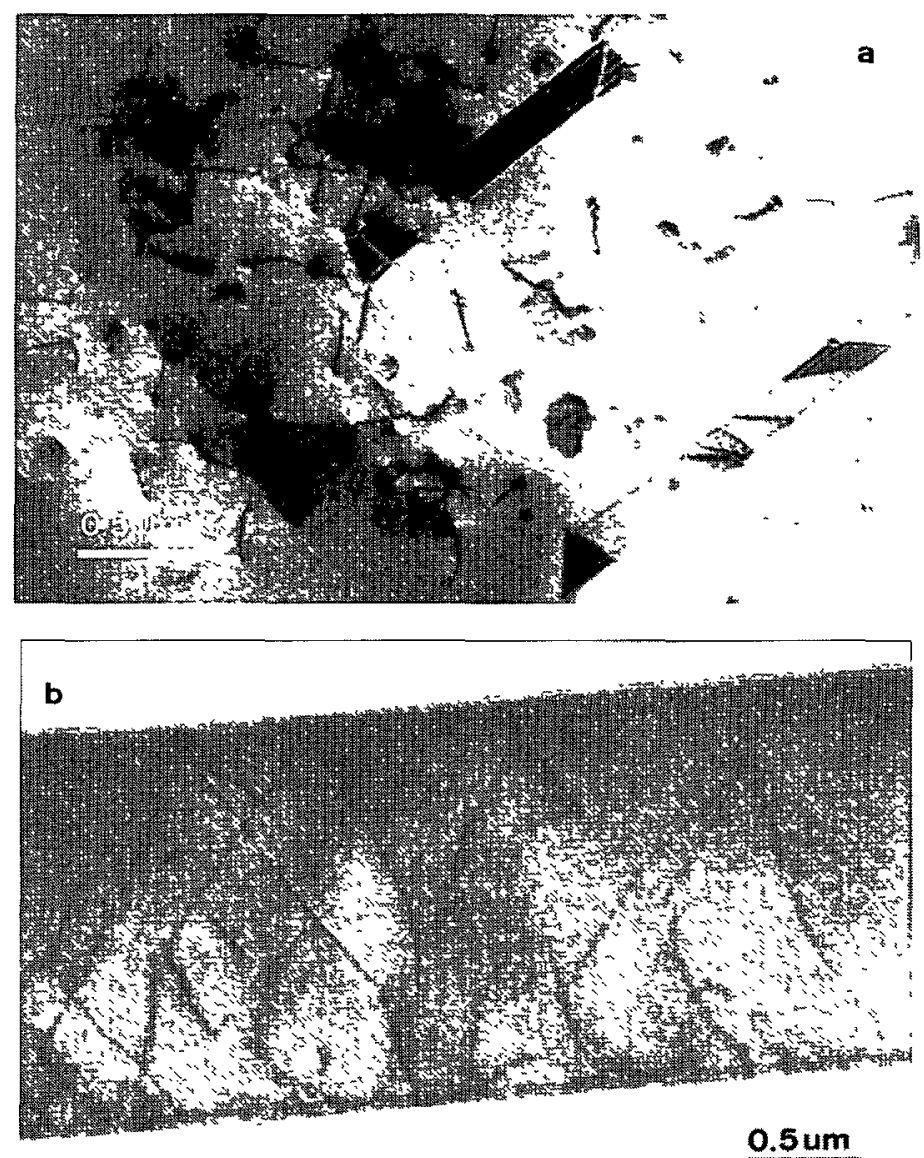

Fig 1 - TEM plan view (a) and cross section (b) of A2 sample. Stacking faults and threading dislocations are the main type of defects.

JEOL JSM 840 measurements were performed in order to study the effect of three-dimensional defects on surface morphology. Raman spectroscopy measurements were performed using a T64000 Jovin-Yvon triple spectrometer, with a $\lambda=541 \mathrm{~nm}$ laser in backscattering configuration.

\section{Results}

Several types of defects have been found in the InSb layers. The type of defects prevailing in all $\mathrm{A}$ specimens are threading dislocations and stacking faults as can be seen in Figure la. The density of threading dislocations is higher than the density of stacking faults (see Tab. I). The stacking faults are usually found in groups developing squared shapes. Calculations based on the size of these groups show that the stacking faults have a common origin at the interface. No anisotropy in defect distribution is found in this series.

According to previous reports on MBE growth of InSb on GaAs [1] the defect densities are higher in a region near the interface than in the bulk. In our case it is observed that they 


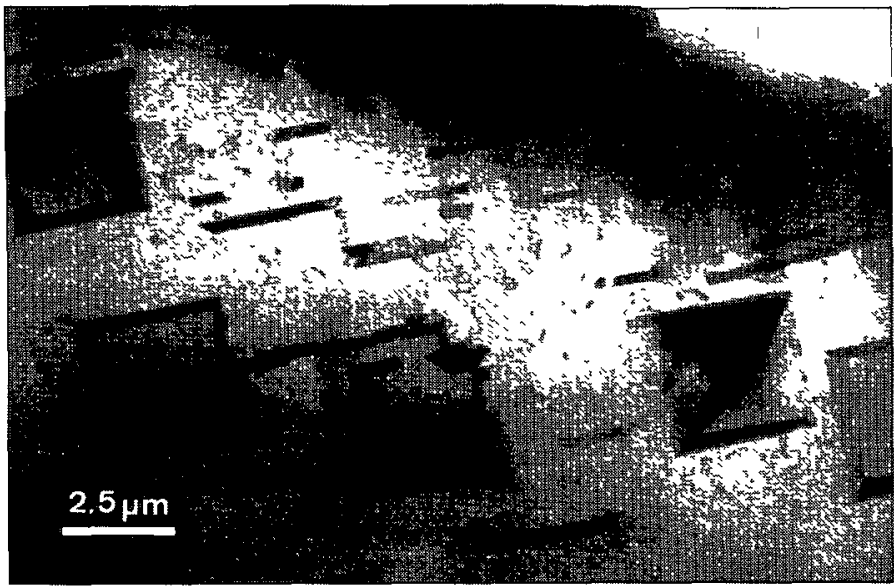

Fig. 2 - TEM plan view of B sample. Besides the threading dislocations and stacking faults, a new type of defects with squared shapes appears.

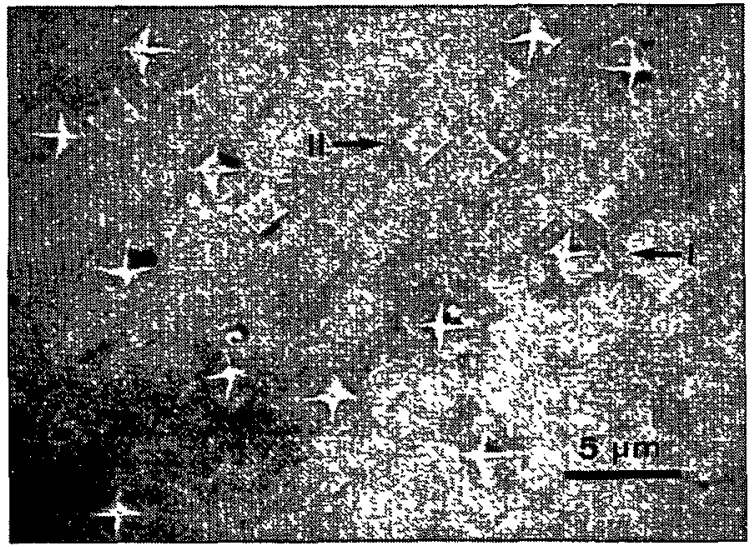

Fig. 3 - SEM ımage of sample B. Pyramidal (I-type defect) and truncated pyramidal hillocks (II-type defect) appear on the surface.

are mainly concentrated in a zone of about $25 \mathrm{~nm}$, where a significant amount of dislocation half-loops are found (Fig. 1b), and that the defect densities at the surface are lower for the thicker sample (A1) compared to the rest of the series.

The sample B, with MBE buffer layers, presents some differences when it is observed with TEM as can be seen in plan view configuration (Fig. 2). Whereas the threading dislocation density is lower than in the preceding cases with similar thickness, the stacking fault density is higher and anisotropic ?.e. higher fault density in the [1]0]-(111)A slip planes than in the $[110]-(111) B$ ones.

Besides the quantitative differences in defect densities, the growth of the buffer layers introduces two new types of defects, that appear as squared structures in plan view TEM images (Fig. 2). Such defects modify the morphology of the sample surface, introducing pyramidal hillocks (I-type defect) or truncated pyramidal hillocks (II-type defect) as can be seen in SEM 


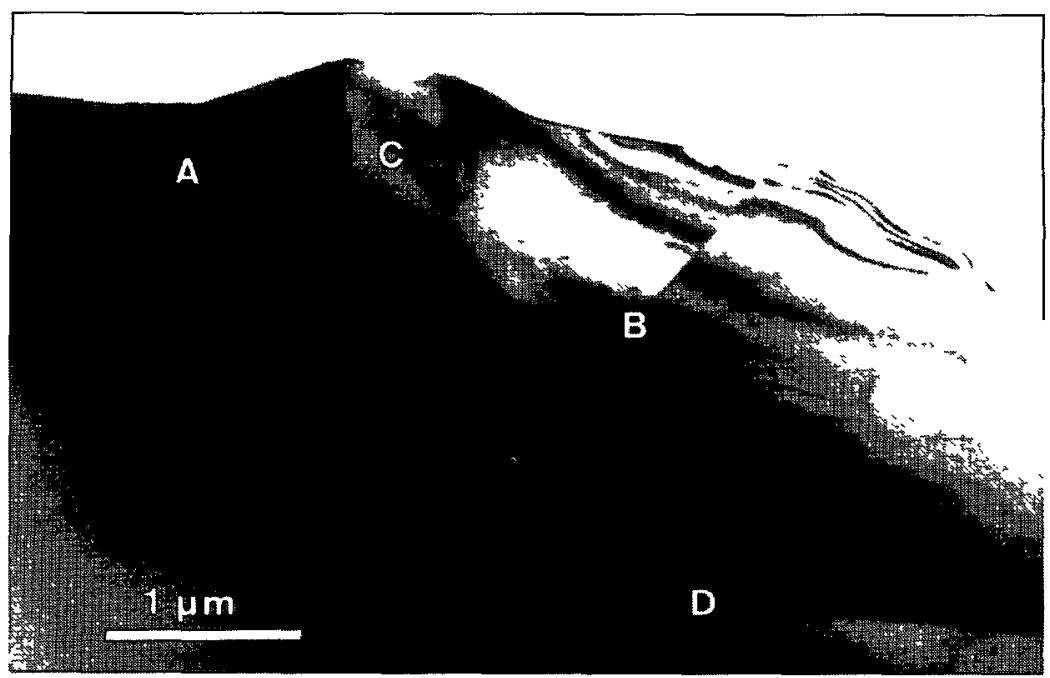

A

\section{C}

B

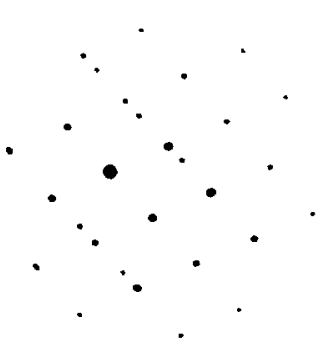

D

Fig 4. - Cross sectional TEM image of a pyramidal hillock Notice that it originates at the InP/InSb MBE interface. Diffraction patterns have been taken at $A, B, C$ and D regions, showing the typical twin-type diffraction (A and B) at the structure boundaries, a $\{114\}$ zone axis diffraction (C) corresponding to a section of a $90^{\circ}$ rotated twined crystal, and a diffraction of the substrate and the InSb layer showing spot splitting because of the relaxed state of the layer, i.e. presence of two different lattice parameters but same lattice structure 


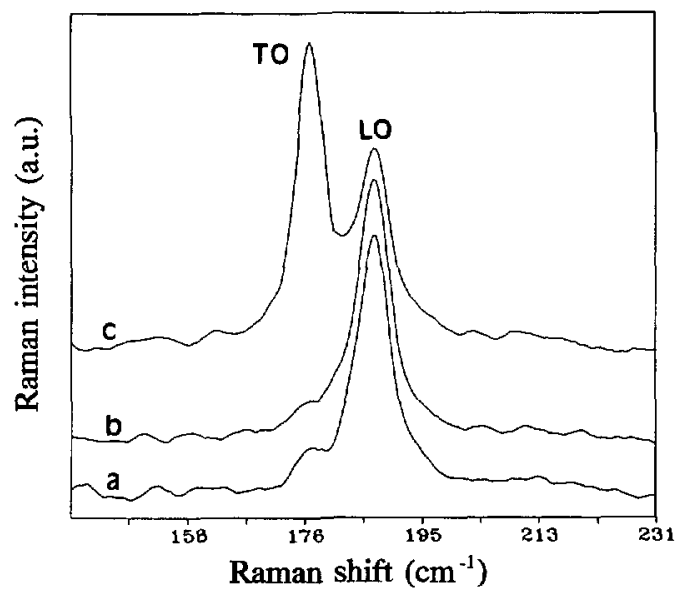

Fig 5. - Raman spectra of B sample at a region free of three-dimensional defects (a), at the centre of a truncated pyramid (b), and at a whole pyramid (c)

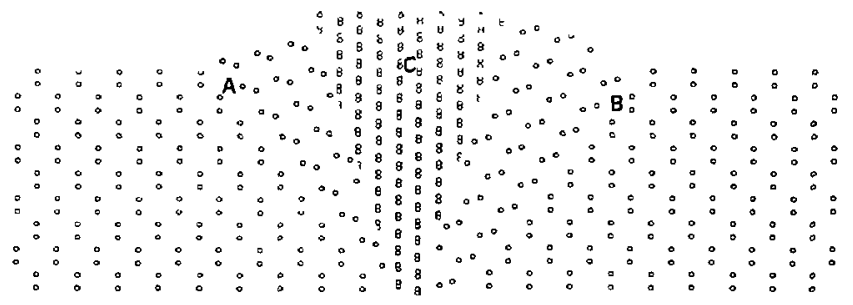

Fig 6. - Ideal structure projection of a pyramidal hillock, showing three of the four twinned crystals Regions $\mathrm{A}, \mathrm{B}$ and $\mathrm{C}$ are the equivalents to Figure 4

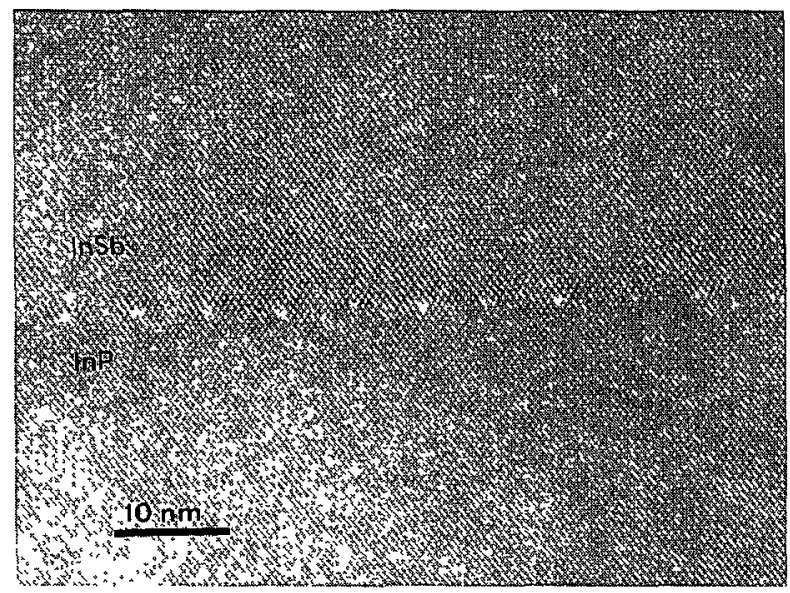

Fig. 7. - High resolution image of the $\mathrm{InP} / \mathrm{InSb}$ interface showing the $90^{\circ}$ dislocation array The mean distance between dislocations is that which relaxes completely the system 
images (Fig. 3). The internal structure of both types of defects may be assessed from the cross-section TEM images and selected area diffraction patterns (SADP) of Figure 4, and the Raman spectra (Fig. 5).

It can be seen in Figure 4 that pyramidal hillocks have their origin at a point placed at the InP/InSb MBE interface where $\Sigma 3$ boundaries contained in $\{111\}$ planes are generated. Two SADP's were taken in the regions overlapping these boundaries showing the typical twin diffraction pattern. Between the two twinned crystals there is another region with a diffraction pattern corresponding to a $\langle 114\rangle$ zone axis, which is just the direction obtained if one of the flanking crystals is rotated $90^{\circ}$ around the [001] direction Thus, the pyramidal hillocks are composed by four twinned crystals, with a common origin, each one corresponding to a pyramid face, which are induced by the different growth rates associated with the new orientations.

The other set of three-dimensional defects, truncated pyramids, have the same origin at the InP/InSb MBE interface. multıple twinning that results in the four lateral sides as in the previous case. However when InSb growth is changed from MBE to ALMBE, the original crystal orientation is recovered between the four crystal misorientations, growing at normal rate and giving the top plane side of this structure. These interpretations agree well with the Raman spectra presented in Figure 5 which were taken at different points of B sample. (a) spectrum was measured in a region free of three-dimensional defects, while (b) and (c) spectra were taken at the centre of a truncated pyramid and at the centre of a whole pyramid respectively. While (a) and (b) spectra are qualitatively the same, which agree well with the idea that the crystalline orientations inside the truncated pyramid and in the rest of the layer are the same, (c) spectrum has the TO mode activated due to a change in crystal orientation.

In order to aid to understand the internal structure of these defects, an ideal structure of pyramidal defects as derived from the TEM images and diffraction patterns is presented in Figure 6.

It is well known that intermediate buffer layers improve the crystalline quality of the upper grown layers. Nevertheless, in our case, the intermediate buffer has been grown by the conventional MBE technique. Our results corroborate that the ALMBE technique favours the surface atomic migration leading to better $\operatorname{InSb} / \operatorname{InP}$ interface $[8,9]$.

Finally, the HRTEM observations of the interface region show that for all samples, there is a regularly distributed array of $90^{\circ}$ dislocations with both dislocation line and Burgers' vector $(a / 2)\langle 110\rangle$ remaining in the interface (see Fig. 7). The mean separation between contiguous dislocations is approximately $4.5 \mathrm{~nm}$ which is close to the distance that relaxes completely the system. This is confirmed by the high spot splitting of the diffraction patterns, as it is shown in Figure 4D taken from InP substrate and InSb layer simultaneously. It is usual to find these edge-type dislocation networks in highly mismatched systems such as InSb on GaAs [2] or InAs on $\mathrm{GaP}[10]$ since it is known that they relieve the strain more efficiently than $60^{\circ}$ dislocation arrays. The nucleation mechanism for the pure-edge type depends on the growth mode and the critical thickness for coherent growth, as it is explained in the work of Kiely et al. [2]. In this paper they suggest the possibility that the dislocations could be generated at the borders of InSb islands nucleated at the earliest growth stages because the low critical thickness of these highly mismatched systems. Other mechanisms have been suggested to explain the generation of such dislocations in three dimensional islands, which is, in fact, the first growth mode of our layers as explained in the experimental details. Recently, Chen et al. [11] have proposed one to account for the formation of $90^{\circ}$ type misfit dislocation based upon the nucleation of a Frank partial dislocation at the edges of the dot followed by a stacking fault extended during the growth of the island up to the nucleation of the Shockley partial whose interaction with the Frank one would annihilate the staking fault and give rise to the Lomer dislocation at the interface. 


\section{Conclusions}

In summary, we have analyzed the crystalline morphology of InSb layers grown by ALMBE on (001) InP substrates. Good crystallinity quality is found for samples without buffer layers grown at low temperatures. In this case, threading dislocations are found to be concentrated in a region near the interface in the form of half loops and low stacking fault densities are found. Moreover it is found that strain is efficiently relieved by a regular edge-type misfit dislocation network. Whereas the growth of an intermediate MBE buffer layer leads to an anomalous growth that introduces complex three-dimensional defects consisting in the association of four twins with $\Sigma 3$ boundaries contained in $\{111\}$ planes, it has been demonstrated that the ALMBE technique leads to a better InSb/InP interface. The original orientation may be recovered inside the defects leading to two types of structures that induce pyramidal or truncated pyramidal hillocks on the surface.

\section{Acknowledgments}

This work was funded by the Spanish CICYT program MAT95-0966.

\section{References}

[1] Williams G.M., Cullis A.G., McConville C F., Withehouse C.R. and Smith P.W , TEM studies of the structure of MBE InSb on GaAs, Inst. Phys. Conf. Ser. 100 (1989) 205.

[2] Kiely C.J., Chyi J-I., Rockett A. and Morkoç H., On the microstructure and interfacial structure of InSb layer grown on GaAs(100) by molecular beam epitaxy, Phrlos. Mag. A 60 (1989) 321.

[3] Oh J.E., Bhattacharya P.K., Chen Y C. and Tsukamoto S., Molecular-beam epitaxial growth of high-quality InSb on InP and GaAs substrates, J. Appl. Phys. 66 (1989) 3618.

[4] Parker S.D , Williams R.L., Droopad R., Stradling R.A., BarnhamK.W.J , Holmes S.N., Laverty J., Phillips C.C., Skuras E., Thomas R., Zhang X., Staton-Bevan A. and Pashley D.W., Observation and control of the amphoteric behaviour of Si-doped InSb grown on GaAs by MBE, Semicond. Scr. Technol. 4 (1989) 663.

[5] Rajan K., Gong R. and Webb J., Elastic accomodation of heteroepitaxial InSb films on GaAs, Appl. Phys. Lett. 57 (1990) 1446.

[6] Utzmeier T., Sendra J.R., Anguita J. and Briones F., Resonant raman scattering study of InSb etched by reactive ion beam etching, VIII European Workshop on Molecular Beam Epitaxy, Sierra Nevada (22-24 March 1995) Spain.

[7] Postigo P.A.. Dotor M.L., Golmayo D. and Briones F., Growth of InP at low temperature by Atomic Layer Molecular Beam Epitaxy: control of phosphorus incorporation by Reflectance Difference technique, VIII European Workshop on Molecular Beam Epitaxy, Sierra Nevada (22-24 March 1995) Spain.

[8] González Y., González L., Briones F.. Vilà A., Cornet A. and Morante J R., J. Cryst. Growth 183 (1992) 385.

[9] Brones F. and Ruiz A., Atomic Layer Molecular Beam Epitaxy (ALMBE)· growth kinetics and applications, J. Cryst. Growth 111 (1991) 194.

[10] Chang J.C P., Chin T.P. and Woodall J.M., Incoherent interface of InAs grown directly on GaP(001), Appl. Phys. Lett. 69 (1996) 981.

[11] Chen Y., Lin X.W., Liliental-Weber Z., Washburn J., Klem J.F. and Tsao J.Y., Appl. Phys. Lett. 68 (1996) 111. 